\begin{tabular}{|c|c|c|c|c|c|}
\hline \multirow{2}{*}{$\begin{array}{l}\text { Fraction } \\
\text { number. }\end{array}$} & \multirow{2}{*}{$\begin{array}{c}\text { Ampere } \\
\text { hours. }\end{array}$} & \multicolumn{2}{|c|}{$\begin{array}{r}\text { TABLE IV. } \\
\text { Wt. in } \mathbf{g} .\end{array}$} & \multicolumn{2}{|c|}{ Per cent. } \\
\hline & & $\mathrm{ThO}_{\mathrm{s}}$. & $\mathrm{R}_{2} \mathrm{O}_{2}$ & $\mathrm{ThO}_{2}$. & $\overline{\mathrm{R}_{\mathrm{s}} \mathrm{O}_{\mathrm{s}}}$ \\
\hline I. . . & 3 & 1.53 & I . 44 & 52 & 48 \\
\hline
\end{tabular}

From these results it is seen that a single fractional electrolysis of three hours' duration precipitates nearly $26 \%$ of the thorium present and that the precipitated hydroxides contain $52 \%$ of $\mathrm{ThO}_{2}$ as against $5 \%$ in the original mixture.

ITHACA, N. Y.

[CONTRIBUtion FROM the LABORATORY OF INORganic Chemistry, MassachusetTs INSTITUTE OF TECHNOLOGY.]

\title{
THE PRECIPITATION OF COLLOIDAL GOLD AND PLATINUM ON METALLIC SURFACES.
}

By Elliwood B. SPEAR aNd KenNeTH D. KaHN.

Received November 6, 1917.

In this article we submit experimental evidence to show that when colloidal gold or platinum is precipitated by metallic surfaces the most active metals are the most effective, that is, the more electropositive the metal is toward the hydrogen electrode the shorter will be the time required for the complete precipitation of the colloid. The evidence also shows that some of the metal, of which the surface is composed dissolves, is adsorbed by the colloidal particles, and is consequently carried down with the colloid. The precipitation of the colloid by metallic plates is therefore quite analogous to that produced by the addition of an electrolyte.

Our attention was directed to this work through an article by $\mathrm{M}$. Phillippson ${ }^{1}$ in which he stated that colloidal silver is precipitated by zinc plates but not by plates of gold or platinum. Phillippson was unable to form any definite conclusions relative to the mechanism of the precipitation, although he surmised that the position of the precipitating agent in the electrode potential series was a vital factor. This we have shown to be correct. Phillippson also suggested that the relation of the activity of the precipitating metal to that of the colloidal metal might have a deciding influence. For example, because copper is more active than silver a plate of copper could precipitate colloidal silver, but a silver plate could not precipitate colloidal copper. From the results of our investigations there would seem to be no necessity for making such an assumption in order to explain the precipitation.

\section{Preparation of the Colloidal Solutions.}

The water used in the preparation of the colloidal solutions was that employed for conductivity purposes in the Research Laboratory of

${ }^{1}$ Kolloid Z., II, 49 (1912). 
Physical Chemistry of the Institute. The colloidal solutions of gold and platinum were prepared by Bredig's well-known method of electrical colloidation. No alkali was added while producing solutions of platinum, but because of its instability in pure water, colloidal gold was made in o.oor $N$ sodium hydroxide solutions.

All the vessels employed were carefully cleansed with chromate solutions, thoroughly washed with distilled water, rinsed with conductivity water and finally treated with steam from conductivity water. These precautions insure the stability of the colloidal solutions, some of which have been kept in the laboratory for over two years without any appreciable amount of precipitation taking place.

\section{Experimental Method.}

The first experimental work was designed to determine the relation of the precipitating effect of the metal and its position in the electrode potential series. To this end well-polished plates of zinc, steel, nickel, lead, tin, copper and platinum having approximately the same amount of exposed surface, were placed in a definite amount of the gold and platinum solutions. All conditions as to temperature, amount of surface of the liquid exposed to the air, etc., were kept as nearly as possible identical throughout each series. Table I gives a typical set of results for colloidal platinum, while Table II gives a similar set for colloidal gold.

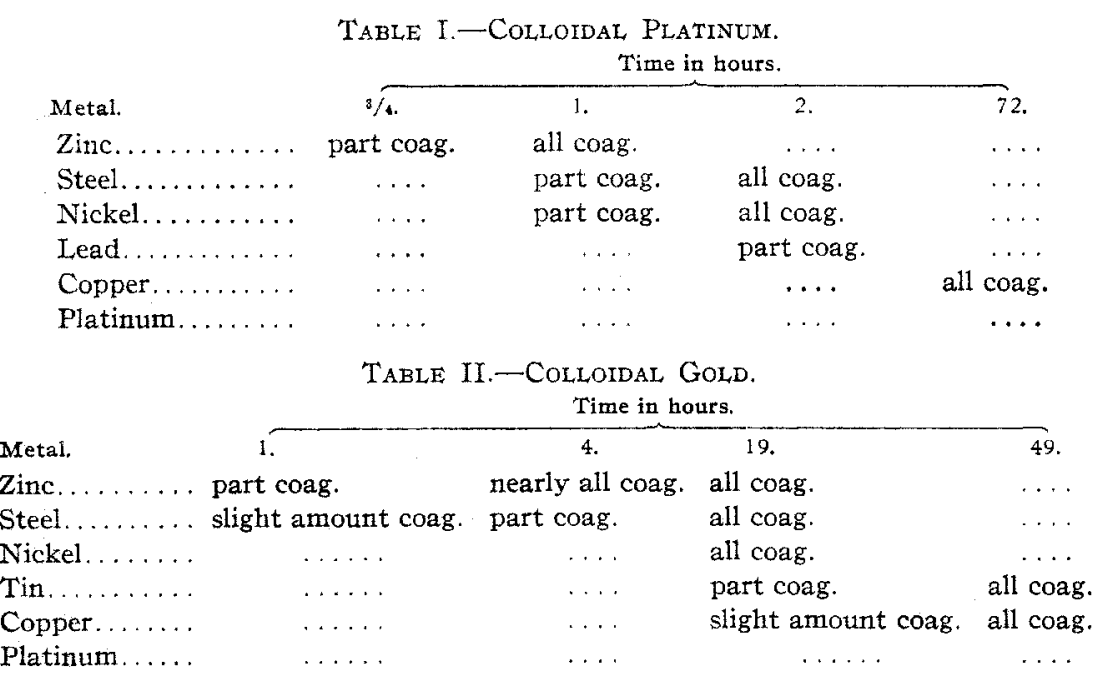

It is evident from the above results that the most reactive metals require the least time to coagulate the colloidal solutions and that inactive metals will not produce coagulation at all. It should be noted, however, that the condition of the surface is an important factor. For instance, it was found that slightly rough surfaces formed by the electrolytic de- 
position of the metal were more efficient than smooth plain surfaces. Thus a copper strip having a plain surface had no visible effect on a quantity of colloidal gold in 24 hours, while another portion of the same strip, the surface of which had been roughened by electroplating, caused complete precipitation in this time. The same accelerated effect of the electrolytic over the plain surface was noted in the case of nickel and also that of lead. Moreover, as one might anticipate, it was found that zinc dust would precipitate colloidal gold or platinum much more rapidly than the same weight of zinc in the plate form. In fact $2 \mathrm{~g}$. of zinc dust gently shaken with to $\mathrm{cc}$. of platinum solution caused complete precipitation in less than one minute.

From a consideration of these results it would seem that the cause of the precipitation is the dissolving of the metal to form the corresponding positively charged ion. The ion then neutralizes the negative charge on the particles and consequently the colloid coagulates.

If this theory is correct it should be possible to prevent a copper plate from precipitating the colloid by keeping out the air because copper does not dissolve in pure water. In fact copper will not even tarnish in water from which the oxygen has been driven by hydrogen. That oxygen is essential for the precipitation of the colloid by copper was shown in the following manner:

A test tube containing the colloidal platinum was fitted with a twohole stopper, through which two glass tubes passed. One of the tubes reached to the bottom of the test tube and served to introduce purified hydrogen into the solution. The other tube allowed hydrogen and the air to escape. The gas was bubbled through the solution for one-half hour, at the end of which time a plate of copper was introduced. Hydrogen was again bubbled through for one hour after which the tube was closed by means of pinchcocks. Another portion of the same copper plate was put into the same amount of the colloidal solution and the container left open to the air. The colloid was coagulated in the open tube in one experiment in 44 hours and in another in 72 hours, while the copper plates in the atmosphere of hydrogen had not affected the colloid in six days. When the tubes containing the hydrogen were opened to the air the colloid was completely coagulated in less than three days. This shows quite clearly that the metal must dissolve before precipitation can take place.

We were also able to predict another result from a consideration of this theory. If the metal dissolves on the surface, forms the ion and the ion discharges the colloidal particles, then, reasoning from our knowledge of precipitation by electrolytes, some of the metal should be adsorbed and carried down by the precipitated colloid. After several unsuccessful 
attempts with lead and copper, due to the relatively small amounts of the colloid used, we were able to show that copper is carried down with colloidal platinum when the precipitation is achieved by means of copper wires. The determination was made as follows:

$600 \mathrm{cc}$. of a concentrated solution of colloidal platinum were placed in a beaker of one-liter capacity. Copper wires of about $1.5 \mathrm{~mm}$. diameter were heated and reduced with alcohol. All tarnished portions were cut off and discarded. The wires were weighed, placed in the solution and the latter stirred at frequent intervals. The precipitation was complete in 36 hours. The copper wires were tarnished and, as might be expected, had increased slightly in weight. The precipitate was filtered and thoroughly washed with conductivity water. Neither the filtrate nor the wash water gave the slightest coloration with hydrogen sulfide.

The precipitate was treated in a casserole with concentrated nitric acid, evaporated to a small volume, diluted with water and filtered. The filtrate which was slightly brown was evaporated to about $2 \mathrm{cc}$. in order to drive off the excess of acid. It was then diluted with ro $\mathrm{cc}$. distilled water and saturated with hydrogen sulfide. A very considerable precipitate was obtained showing that coagulated colloidal platinum is appreciably attacked by concentrated nitric acid. The precipitated sulfides were then treated with ammonium polysulfide, whereby most or all of the platinum sulfide dissolved. The filter paper was washed and boiled with $2 N$ nitric acid. After filtering, the liquid was evaporated to a small volume, diluted with water, neutralized with ammonia and acidified with acetic acid. Two cc. of a solution of potassium ferrocyanide were then added. The first drop gave an unmistakable copper reaction. After standing some hours a small pink precipitate settled to the bottom of the tube. These experiments prove beyond a doubt that copper dissolves and is carried down by the colloid.

\section{Summary.}

In this article we have shown that:

Metallic plates precipitate colloidal platinum and gold in the same order as the metals occur in the electrode potential series, the most active metal requiring the least time.

A roughened surface or a finely divided metal requires less time than the same metal if the surface is smooth.

The metal dissolves, is adsorbed and carried down by the colloid.

The precipitating effect of the metal may be explained on the same basis as that of electrolytes.

Cambridge, Mass. 